\title{
Solutions of reaction-diffusion equations using similarity reduction and HSSOR iteration
}

\author{
Nur Afza Mat Ali, Rostang Rahman, Jumat Sulaiman, Khadizah Ghazali
}

Faculty of Science and Natural Resources, Universiti Malaysia Sabah (UMS), Malaysia

\begin{tabular}{l}
\hline \hline Article Info \\
\hline Article history: \\
Received Dec 30, 2018 \\
Revised Apr 23, 2019 \\
Accepted Jun 28, 2019 \\
\hline
\end{tabular}

\section{Keywords:}

Finite difference

HSSOR iteration

Reaction-diffusion equation

Wave variable transformation

\begin{abstract}
Similarity method is used in finding the solutions of partial differential equation (PDE) in reduction to the corresponding ordinary differential equation (ODE) which are not easily integrable in terms of elementary or tabulated functions. Then, the Half-Sweep Successive Over-Relaxation (HSSOR) iterative method is applied in solving the sparse linear system which is generated from the discretization process of the corresponding second order ODEs with Dirichlet boundary conditions. Basically, this ODEs has been constructed from one-dimensional reaction-diffusion equations by using wave variable transformation. Having a large-scale and sparse linear system, we conduct the performances analysis of three iterative methods such as Full-sweep Gauss-Seidel (FSGS), Full-sweep Successive Over-Relaxation (FSSOR) and HSSOR iterative methods to examine the effectiveness of their computational cost. Therefore, four examples of these problems were tested to observe the performance of the proposed iterative methods. Throughout implementation of numerical experiments, three parameters have been considered which are number of iterations, execution time and maximum absolute error. According to the numerical results, the HSSOR method is the most efficient iterative method in solving the proposed problem with the least number of iterations and execution time followed by FSSOR and FSGS iterative methods.
\end{abstract}

Copyright $@ 2019$ Institute of Advanced Engineering and Science. All rights reserved.

\section{Corresponding Author:}

Nur Afza Mat Ali,

Faculty of Science and Natural Resources, Universiti Malaysia Sabah, Sabah, Malaysia.

Email: afzamatali@yahoo.com

\section{INTRODUCTION}

The problem of parabolic PDEs are very important to be solved numerically since this problem will be applied in variety of application including in branches of science and engineering [1-3]. Therefore, it is clearly showed that development of an efficient and reliable method is very needed in tackling this issue. Actually, it is necessary to use the numerical approach in many application parts to get an approximate solution of one-dimensional reaction-diffusion equation. In the similar context, several numerical methods such as Hopf Bifurcation [4] and Method of Moments [5] have been proposed to solve particularly on mathematical models based on differential equations. Apart from the above numerical methods, the use of the discretization process via the two-level [6] or three-level scheme [7] of finite difference method particularly an implicit or Crank-Nicolson has been imposed to discretize the mathematical models and then get the corresponding approximation equation. It can be observed that this approximation equation leads a sequence of corresponding linear systems at each time level. It means that these linear systems need to be solved iteratively or directly. Directly, the computational time of getting an approximate solution will be increasing due to their high computational complexity. To avoid this matter, this study has proposed the similarity reduction method reduce the computational complexity during the discretization process in order to form a single similarity linear system. 
Since having the similarity linear system as mentioned in the first paragraph, we focus on using Successive Over Relaxation (SOR) method combined with Half-Sweep (HS) concept whereas this iterative method is very useful in order to solve sparse linear systems [8]. Based on the previous studies of solving linear systems conducted by Young [9] as well as Hackbusch [10], they pointed out that SOR method is one of the effective point iterative methods in solving a large scale and sparse linear system. Due to its advantage of using SOR iteration, the simulation process will converge faster in which their execution time and number of iterations get smaller in solving the one-dimensional reaction-diffusion equation as compared to GS method.

In this paper, the main objective is to deal with effectiveness of HSSOR iterative method with the similarity reduction in solving one-dimensional reaction-diffusion equation. To examine the effectiveness of this combination, let us consider the one-dimensional reaction-diffusion equation as follows.

$$
\frac{\partial u}{\partial t}=\alpha \frac{\partial^{2} u}{\partial t^{2}}+p(t) u+\phi(x, t), 0 \leq x \leq n, 0<t \leq T
$$

with the initial condition

$$
u(x, 0)=f(x), 0 \leq x \leq n
$$

and the boundary conditions

$$
\begin{array}{ll}
u(0, t)=g_{0}(t), & 0<t \leq T \\
u(n, t)=g_{1}(t), & 0<t \leq T
\end{array}
$$

where $f, g_{0}, g_{1}, \phi, E$ and $\alpha$ are known, while the functions $u$ need to be determined. In order to develop an approximation equation of problem (1), let length of $[0, n]$ for derivation of the $n$ subinterval over the original internal $[0, n]$ finite difference scheme be given as follows

$$
\Delta x=\frac{(n-0)}{n}=h,
$$

Based on equation (2), the distribution of uniformly grid points over the solution domain $[0, n]$ is illustrated in Figure 1

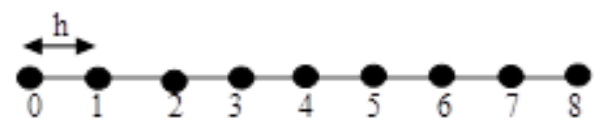

Figure 1. Finite grid network for solution domain at $n=8$

For the next discussion, this paper is organized as follows. In section 2, discretization of the similarity problem which is derived by problem (1) is carried out by using second-order central finite difference schemes to generate a linear system. In section 3, we show the formulation of HSSOR in order to solve the corresponding linear system. Then, four tested numerical results and their discussion are given in section 4. Finally, in section 5 we give some conclusions and future work for this problem.

\section{SIMILARITY REDUCTION VIA HALF-SWEEP FINITE DIFFERENCE SCHEME}

In this section, a linear system is generated using a finite difference approximate equation. Before having this linear system, the similarities reduction method will be imposed into problem (1) which means that PDE (1) will be reduced to an ODE in order to form only a linear system. In addition to do that, there are various techniques for PDE to be reduced to an ODE include various integral transform and eigenfunction expansions [11]. However, this study use the similarities reduction for PDE namely wave variable transformation technique. The essential idea of this procedure is to seek solutions of the form $u(x, t)$ 
becomes $u(\xi)$ where $\xi=(x-c t)$ is called as wave variable [12,13]. To apply this reduction, the general linear PDE is considered and given as follows

$$
J\left(u, u_{x}, u_{t}, u_{x x}, u_{t t}, \ldots\right)=0
$$

The following equation (4) shows the general linear ODE after applying wave variable transform

$$
K\left(u, u_{\xi}, u_{\xi \xi}, \ldots\right)=0
$$

Before discretizing the proposed problem in equation (1), let us consider the following terms of wave variable transformation to reduce the variables in equation (1) as follows

$$
\left.\begin{array}{l}
\frac{\partial}{\partial t}=-c \frac{\partial}{\partial \xi}, \frac{\partial^{2}}{\partial t^{2}}=c^{2} \frac{\partial^{2}}{\partial \xi^{2}} \\
\frac{\partial}{\partial x}=\frac{\partial}{\partial \xi}, \frac{\partial^{2}}{\partial x^{2}}=\frac{\partial^{2}}{\partial \xi^{2}} .
\end{array}\right\}
$$

By using the corresponding terms (5), problem (1) can be easily transformed into the linear ODE and state as follows

$$
\alpha \frac{\partial^{2} u}{\partial \xi^{2}}+c \frac{\partial u}{\partial \xi}+p(\xi) u(\xi)=-\phi(\xi)
$$

Figure 2 shows the implementation of the Full-sweep (FS) and Half-sweep (HS) iterations that will compute the approximate values of interior node points of type only until the convergence criterion is reached. Meanwhile, the node points type $\bigcirc$ shows the other approximate solutions at remaining points which solving by using direct methods [14].

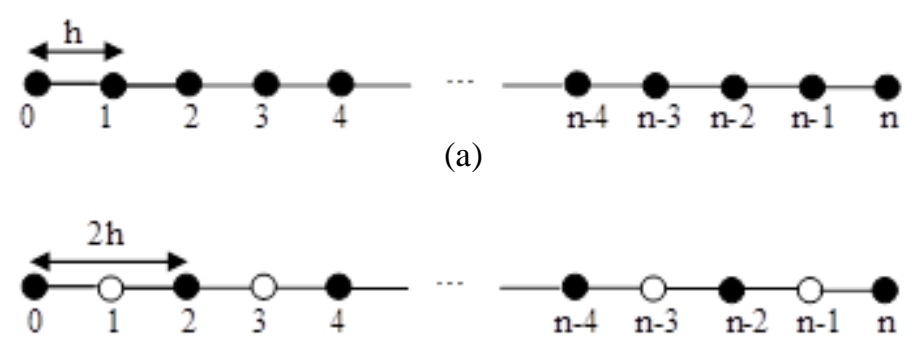

(b)

Figure 2. (a) and (b) represent the illustration of finite grid network for FS and HS iteration respectively

By considering the distribution of node points in Figure 1, Figure 2(a) and Figure 2(b) represent the illustration of finite grid network for FS and HS iteration respectively. According to Figure 2, it seems that the implementation of the HS iteration just involves half of whole inner node points as shown in Figure 2(b) compared with the FS iteration as shown in Figure 2(a). Then, in order to construct the generated linear system, let the corresponding ODE (6) be discretized by applying the second-order HS central finite difference scheme to form the corresponding approximation equation. Before discretizing equation (6), let us consider the following second-order HS central finite difference schemes [14]. 


$$
\left.\begin{array}{l}
\left.\frac{d u}{d \xi}\right|_{i}=\frac{u_{i+2}-u_{i-2}}{4 h} \\
\left.\frac{d^{2} u}{d \xi^{2}}\right|_{i}=\frac{u_{i+2}-2 u_{i}+u_{i-2}}{4 h^{2}}
\end{array}\right\}
$$

Thus, by imposing the second-order HS central difference scheme in equation (7), the second-order HS finite difference approximation equation for problem (6) can be formed as follows

$$
\alpha\left(\frac{u_{i+2}-2 u_{i}+u_{i-2}}{4 h^{2}}\right)+c\left(\frac{u_{i+2}-u_{i-2}}{4 h}\right)+p_{i}\left(u_{i}\right)=-\phi_{i}
$$

For convenience, we simplify equation (8) and rewrite it in the following equation

$$
A u_{i-2}+B_{i} u_{i}+C u_{i+2}=-\phi_{i}, \quad i=2,4,6, \ldots, n-2
$$

where

$$
A=\frac{\alpha}{4 h^{2}}-\frac{c}{4 h}, B_{i}=p_{i}-\frac{\alpha}{2 h^{2}}, C=\frac{\alpha}{4 h^{2}}+\frac{c}{4 h}
$$

The following linear system generated using finite difference method can be easily show $n$ in matrix form as follows

$$
W \underline{U}=\underline{\phi}
$$

where

$$
W=\left[\begin{array}{cccccc}
B_{2} & C & & & & \\
A & B_{4} & C & & & \\
& A & B_{6} & C & & \\
& & \ddots & \ddots & \ddots & \\
& & & A & B_{n-4} & C \\
& & & & A & B_{n-2}
\end{array}\right] \quad \underline{U}=\left[\begin{array}{c}
U_{2} \\
U_{4} \\
U_{6} \\
\vdots \\
U_{n-4} \\
U_{n-2}
\end{array}\right] \quad \underline{\phi}=\left[\begin{array}{c}
-A U_{0}-\phi_{2} \\
\phi_{4} \\
\phi_{6} \\
\vdots \\
\phi_{n-4} \\
-C U_{n}-\phi_{n-2}
\end{array}\right] .
$$

According to linear system (10), it can be revealed that the coefficient matrix A is categorized as large-scale and sparse matrix. Due to get the similarity solution of linear system (10), the iterative method is the key to the solution of the linear system. Therefore, we consider the iterative methods such as HSSOR, FSSOR and FSGS in solving the linear system (10) as explained in the next section.

\section{FORMULATION OF HALF-SWEEP SOR ITERATION}

As mentioned in section 2, the iterative method namely SOR is applied with combination of HS concept to solve the sparse linear system (10). The HS iteration concept is known as the complexity reduction approach [15] which is introduced by Abdullah [16]. Due to its advantage, many authors have been attracted to use the applications of the HS iteration concept in solving the numerical issues in their research [17-20]. Therefore, the corresponding sparse linear system (10) also has been solved by using the HS iteration concept via the SOR method since the SOR method is the common option for efficient solution. Based on Hadjidimos [21] and Young [22], they have mentioned that the SOR method is one of the most known and widely used iterative method in solving any system of linear equations.

In order to implement the iterative process, let the formulation of the HSSOR iterative methods be constructed from the approximation equation (9) by considering the following splitting of its coefficient matrix as: 


$$
W=D-L-V
$$

where each matrix of $\mathrm{D}, \mathrm{L}$ and $\mathrm{V}$ can be defined as

$$
D=\left[\begin{array}{cccccc}
B_{2} & 0 & 0 & 0 & 0 & 0 \\
0 & B_{4} & 0 & 0 & 0 & 0 \\
0 & 0 & B_{6} & 0 & 0 & 0 \\
0 & 0 & 0 & \ddots & 0 & 0 \\
0 & 0 & 0 & 0 & B_{n-4} & 0 \\
0 & 0 & 0 & 0 & 0 & B_{n-2}
\end{array}\right], L=\left[\begin{array}{cccccc}
0 & 0 & 0 & 0 & 0 & 0 \\
-A & 0 & 0 & 0 & 0 & 0 \\
0 & -A & 0 & 0 & 0 & 0 \\
0 & 0 & \ddots & 0 & 0 & 0 \\
0 & 0 & 0 & -A & 0 & 0 \\
0 & 0 & 0 & 0 & -A & 0
\end{array}\right], V=\left[\begin{array}{cccccc}
0 & -C & 0 & 0 & 0 & 0 \\
0 & 0 & -C & 0 & 0 & 0 \\
0 & 0 & 0 & -C & 0 & 0 \\
0 & 0 & 0 & 0 & \ddots & 0 \\
0 & 0 & 0 & 0 & 0 & -C \\
0 & 0 & 0 & 0 & 0 & 0
\end{array}\right] .
$$

By manipulating equation (11), it can be showed that the general form of HSSOR iterative methods can be stated as $[18,19]$

$$
\underline{U}^{(k+1)}=(1-\omega) \underline{U}^{(k)}+\omega(D+L)^{-1}\left(-V \underline{U}^{(k)}+\underline{\phi}\right)
$$

Actually, SOR method is generalization and improvement of the Gauss-Seidel (GS) method together with the weighted parameter, $\omega$ in the range of $1 \leq \omega<2$. For $\omega=1$, the SOR becomes the GS method. Weighted parameter can be determined essentially by sequentially selecting a value with some accuracy until the optimal value can be obtained. Based on equation (12), the general algorithm for the implementation of HSSOR iterative method in solving the linear system (10) may be demonstrated in Algorithm 1 [23].

Algorithm 1: HSSOR iteration

i. Set all the parameters.

ii. Assign the optimal value of $\omega$.

iii. Solve the system by calculating the value of using

$$
\underline{U}^{(k+1)}=(1-\omega) \underline{U}^{(k)}+\omega(D+L)^{-1}\left(-V \underline{U}^{(k)}+\underline{\phi}\right) .
$$

iv. Perform the convergence test, $\left|U_{i}{ }^{(k+1)}-U_{i}{ }^{(k)}\right| \leq \varepsilon=10^{-10}$. If yes, move to step (v). Otherwise go to step (iii).

v. Stop.

\section{NUMERICAL RESULTS AND DISCUSSION}

In this section, four numerical tests have been conducted in order to verify the effectiveness of the HSSOR method as compared with the FSSOR and FSGS methods. As a comparison among of these three iterative methods, three criteria including number of iterations, execution time which measured in second and maximum absolute error are considered. Through the numerical simulations, we have set up the tolerance error, $\varepsilon=10^{-10}$ as a convergence test at different sizes grid which are $m=512,1024,2048,4096$ and 8192 . We have used maximum absolute error as stopping error in which it is very helpful when the convergence is very slow to converge. We have tested the proposed methods on the following four examples:

\subsection{Example 1 [24]}

Let us consider the one-dimensional problem as follows

$$
\frac{\partial u}{\partial t}=\alpha \frac{\partial^{2} u}{\partial t^{2}}+\left(1+t^{2}\right)+\left[\pi^{2}-(t+1)^{2}\right] e^{-t^{2}}[\cos (\pi x)+\sin (\pi x)]
$$

with the initial condition

$$
u(x, 0)=f(x), 0 \leq x \leq 1
$$


and boundary conditions

$$
\begin{aligned}
& u(0, t)=g_{0}(t), \quad 0<t \leq T, \\
& u(1, t)=g_{1}(t), \quad 0<t \leq T
\end{aligned}
$$

The analytical solution of problem (13) is

$$
u(x, t)=\exp \left(-t^{2}\right)(\cos (\pi x)+\sin (\pi x))
$$

\subsection{Example 2 [25]}

Consider the following PDE which is found in many transport phenomena

$$
\frac{\partial u}{\partial t}=\frac{\partial^{2} u}{\partial x^{2}}+4-6 x, \quad t>0,0<x<1
$$

with the initial condition

$$
u(x, 0)=\sin \pi x+x(1-x)^{2} \quad 0 \leq x \leq 1
$$

and boundary conditions

$$
u(0, t)=u(1, t)=0
$$

The analytical solution of problem (15) is

$$
u(x, t)=e^{-\pi^{2} t} \sin (\pi x)+x(1-x)^{2}
$$

\subsection{Example 3 [26]}

Consider the following equation

$$
\frac{\partial u}{\partial t}=[2+\cos (t)] \frac{\partial^{2} u}{\partial x^{2}}+[3+\cos (t)] e^{t} \cos (x), \quad(x, t) \in(0,1) \times(0, T]
$$

with the initial condition

$$
u(x, 0)=f(x), x \in[0,1]
$$

and boundary conditions

$$
\begin{aligned}
& u(0, t)=g_{0}(t) t \in[0, T] \\
& u(1, t)=g_{1}(t) t \in[0, T]
\end{aligned}
$$

The analytical solution of problem (17) is

$$
u(x, t)=e^{t} \cos (x)
$$

\subsection{Example 4 [27]}

Consider the following diffusion problem

$$
\frac{\partial u}{\partial t}=\frac{\partial^{2} u}{\partial x^{2}}-u
$$

with the initial condition 


$$
u(x, 0)=x+e^{-x}
$$

While, the analytical solution of problem (19) is

$$
u(x, t)=e^{-x}+x e^{-t}
$$

Table 1 shows the comparison of the three criteria by using three purposed iterative methods in order to examine their performance. Meanwhile, Table 2 represents the percentage of reduction for the HSSOR and FSSOR method as compared to FSGS iterative method.

\begin{tabular}{|c|c|c|c|c|c|c|c|c|c|c|}
\hline \multirow[t]{2}{*}{ EXAMPLE } & \multirow{2}{*}{$\begin{array}{l}\text { GRID } \\
\text { SIZES }\end{array}$} & \multicolumn{3}{|c|}{ ITERATION NUMBER } & \multicolumn{3}{|c|}{$\begin{array}{l}\text { EXECUTION TIME } \\
\text { (SECOND) }\end{array}$} & \multicolumn{3}{|c|}{$\begin{array}{c}\text { MAXIMUM ABSOLUTE } \\
\text { ERROR }\end{array}$} \\
\hline & & FSGS & FSSOR & HSSOR & FSGS & FSSOR & HSSOR & FSGS & FSSOR & HSSOR \\
\hline \multirow{5}{*}{1} & 512 & 193056 & 1617 & 792 & 3.78 & 0.06 & 0.02 & $2.2249 \mathrm{e}-02$ & $2.2252 \mathrm{e}-02$ & $2.2252 \mathrm{e}-02$ \\
\hline & 1024 & 627163 & 3271 & 1617 & 24.48 & 0.16 & 0.07 & $2.2242 \mathrm{e}-02$ & $2.2252 \mathrm{e}-02$ & $2.2252 \mathrm{e}-02$ \\
\hline & 2048 & 1931198 & 6580 & 3271 & 150.44 & 0.59 & 0.20 & $2.2211 \mathrm{e}-02$ & $2.2252 \mathrm{e}-02$ & $2.2252 \mathrm{e}-02$ \\
\hline & 4096 & 5420290 & 17064 & 6580 & 847.91 & 3.02 & 0.63 & $2.2087 \mathrm{e}-02$ & $2.2252 \mathrm{e}-02$ & $2.2252 \mathrm{e}-02$ \\
\hline & 8192 & 12408135 & 32231 & 17064 & 3881.56 & 11.34 & 3.01 & $2.1592 \mathrm{e}-02$ & $2.2252 \mathrm{e}-02$ & $2.2252 \mathrm{e}-02$ \\
\hline \multirow{5}{*}{2} & 512 & 275982 & 1522 & 765 & 5.40 & 0.08 & 0.02 & $1.9675 \mathrm{e}-02$ & $1.9672 \mathrm{e}-02$ & $1.9672 \mathrm{e}-02$ \\
\hline & 1024 & 960413 & 2815 & 1522 & 37.38 & 0.15 & 0.06 & $1.9682 \mathrm{e}-02$ & $1.9672 \mathrm{e}-02$ & $1.9672 \mathrm{e}-02$ \\
\hline & 2048 & 3267333 & 5388 & 2815 & 254.54 & 0.51 & 0.25 & $1.9709 \mathrm{e}-02$ & $1.9672 \mathrm{e}-02$ & $1.9672 \mathrm{e}-02$ \\
\hline & 4096 & 10771529 & 14687 & 5388 & 7076.77 & 2.59 & 0.52 & $1.9819 \mathrm{e}-02$ & $1.9673 \mathrm{e}-02$ & $1.9672 \mathrm{e}-02$ \\
\hline & 8192 & 33893854 & 34560 & 14687 & 10665.73 & 12.10 & 2.61 & $2.0259 \mathrm{e}-02$ & $1.9673 \mathrm{e}-02$ & $1.9673 \mathrm{e}-02$ \\
\hline \multirow{5}{*}{3} & 512 & 368920 & 2049 & 1025 & 8.59 & 0.08 & 0.03 & $5.5183 \mathrm{e}-02$ & $5.5185 \mathrm{e}-02$ & $5.5186 \mathrm{e}-02$ \\
\hline & 1024 & 1329011 & 4097 & 2049 & 61.65 & 0.25 & 0.10 & $5.5175 \mathrm{e}-02$ & $5.5185 \mathrm{e}-02$ & $5.5185 \mathrm{e}-02$ \\
\hline & 2048 & 4729285 & 8193 & 4097 & 439.85 & 0.86 & 0.25 & $5.5145 \mathrm{e}-02$ & $5.5185 \mathrm{e}-02$ & $5.5185 \mathrm{e}-02$ \\
\hline & 4096 & 16569953 & 24155 & 8193 & 3089.34 & 4.95 & 0.88 & $5.5026 \mathrm{e}-02$ & $5.5185 \mathrm{e}-02$ & $5.5185 \mathrm{e}-02$ \\
\hline & 8192 & 56890787 & 46350 & 24155 & 21221.07 & 19.00 & 5.01 & $5.4550 \mathrm{e}-02$ & $5.5185 \mathrm{e}-02$ & $5.5185 \mathrm{e}-02$ \\
\hline \multirow{5}{*}{4} & 512 & 335335 & 2049 & 1025 & 6.49 & 0.08 & 0.02 & $4.5357 \mathrm{e}-02$ & $4.5360 \mathrm{e}-02$ & $4.5360 \mathrm{e}-02$ \\
\hline & 1024 & 1197647 & 4097 & 2049 & 46.24 & 0.20 & 0.07 & $4.5350 \mathrm{e}-02$ & $4.5360 \mathrm{e}-02$ & $4.5360 \mathrm{e}-02$ \\
\hline & 2048 & 4215906 & 8193 & 4097 & 326.38 & 0.71 & 0.21 & $4.5319 \mathrm{e}-02$ & $4.5360 \mathrm{e}-02$ & $4.5360 \mathrm{e}-02$ \\
\hline & 4096 & 14565094 & 22540 & 8193 & 2263.54 & 3.95 & 0.76 & $4.5194 \mathrm{e}-02$ & $4.5360 \mathrm{e}-02$ & $4.5360 \mathrm{e}-02$ \\
\hline & 8192 & 49066665 & 43174 & 22540 & 15239.63 & 15.11 & 3.94 & $4.4697 \mathrm{e}-02$ & $4.5360 \mathrm{e}-02$ & $4.5360 \mathrm{e}-02$ \\
\hline
\end{tabular}

Table 1. Comparison of the three criteria using HSSOR, FSSOR and FSGS iterative methods

According to Table 1, the numerical results of four examples show that the performance of proposed iterative methods consists of number of iterations, execution time in seconds and maximum absolute error. These three parameters have been required to compare the effectiveness of three iterative methods which are HSSOR, FSSOR and FSGS iterative methods in solving the reaction-diffusion equations. As we can see, the combination between SOR method and HS approach has required less number of iterations followed by FSSOR and FSGS methods. Also, we found that the HSSOR method is the fastest iterations process in term of execution time at every grid sizes compared other methods.

Table 2. Percentage reduction for the HSSOR and FSSOR compared to FSGS iterative method

\begin{tabular}{ccccc}
\hline \multirow{2}{*}{ Example } & \multicolumn{2}{c}{ Number of Iterations } & \multicolumn{2}{c}{ Time (second) } \\
& FSSOR & HSSOR & FSSOR & HSSOR \\
\hline 1 & $99.16-99.74$ & $99.59-99.88$ & $98.41-99.71$ & $99.47-99.93$ \\
2 & $99.45-99.90$ & $99.72-99.96$ & $98.52-99.96$ & $99.63-99.99$ \\
3 & $99.44-99.92$ & $99.72-99.96$ & $99.07-99.91$ & $99.65-99.98$ \\
4 & $99.39-99.91$ & $99.69-99.95$ & $98.77-99.90$ & $99.69-99.97$ \\
\hline
\end{tabular}

Based on the performance analysis in Table 2, the percentage of reduction for number of iterations by using HSSOR iterative method is a little bit higher than FSSOR by approximately 99.59-99.96\% and 99.16-99.92\% respectively compared to FSGS iterative method. While in terms of execution time, HSSOR and FSSOR proved to be very faster by approximately $99.47-99.99 \%$ and $98.41-99.96 \%$ respectively compared to FSGS iterative method. It can be concluded that the most efficient iterative method in this study is HSSOR method and followed by FSSOR method. 


\section{CONCLUSION}

This paper presents the similarities solution technique in advantages of forming only a linear system that can be reduced the computational complexity and time during the iteration process. Then, the applications of the HS iteration concept combined with SOR method can be carried out in solving the sparse linear systems that has been generated from corresponding second-order HS central finite difference approximate equation via similarities solution technique. On the whole, the numerical results have showed that HSSOR iterative method is drastically better than FSSOR and FSGS iterative methods due to its smaller number of iterations and execution time. For the future work, we will apply the family of quarter-sweep iteration [28] as linear solvers to solve this problem.

\section{ACKNOWLEDGEMENTS}

This work is supported by the research grant scheme (GUG0225-1/2018) from Universiti Malaysia Sabah, Malaysia for the completion of this paper.

\section{REFERENCES}

[1] J. R. Cannon, et al., "Determination of source parameter in parabolic equations," Meccanica, vol. 27, pp. 85-94, 1992.

[2] J. R. Cannon, et al., "Numerical procedures for the determination of an unknown coefficient in semi-linear parabolic differential equations," Inverse Problems, vol. 10, pp. 227-243, 1994.

[3] M. Zerroukat, et al., "Explicit and implicit meshless methods for linear advection-diffusion type partial differential equations," International Journal for Numerical Methods in Engineering, vol. 48, pp. 19-35, 2000.

[4] G. Zhao and Y. Li, "Hopf Bifurcation in Numerical Approximation for the Generalized Lienard Equation with Finite Delay," TELKOMNIKA Indonesian Journal of Electrical Engineering, vol. 14, pp. 140-146, 2015.

[5] A. H. P. Mohammadi, et al., "Comparison of shielding effectiveness in complex curved structure with different numerical methods, FDTD, MOM and equivalent circuit," Indonesian Journal of Electrical Engineering and Computer Science (IJEECS), vol. 12, pp. 1010-1019, 2018.

[6] J. M. Vaquero and S. Sajavičius, "The two-level finite difference schemes for the heat equation with nonlocal initial condition,” Applied Mathematics and Computation, vol. 342, pp. 166-177, 2019.

[7] I. K. Kaba and W. Dai, "A stable three-level finite difference scheme for solving the parabolic two-step model in a 3D micro-sphere heated by ultrashort-pulsed lasers," Journal of Computational and Applied Mathematics, vol. 181, pp. 125-147, 2005.

[8] A. Saudi and J. Sulaiman, "Path planning for mobile robot with half-sweep successive over-relaxation (HSSOR) iterative method," Proceedings of the Symposium on Progress in Information \& Communication Technology, Kuala Lumpur, pp. 57-62, 2009.

[9] D. M. Young, "Iterative solution of large linear systems," New York, Academic Press, 1971.

[10] W. Hackbusch, "Iterative solution of large sparse systems of equations," First Edition, New York, Springer-Verlag, 1994.

[11] R. Bernatz, "Fourier series and numerical methods for partial differential equations," Hoboken, John Wiley \& Sons, 2010.

[12] A. M. Wazwaz, "The sine-cosine method for handling nonlinear wave equations," Mathematical and Computer Modelling, vol. 40, pp. 499-508, 2004.

[13] S. Bibi and S. T. Mohyud-Din, "Travelling wave solutions of KdVs using sine-cosine method," Journal of the Association of Arab Universities for Basic and Applied Sciences, vol. 15, pp. 90-93, 2014.

[14] J. Sulaiman, et al., "Implicit finite difference solutions of one-dimensional burger's equation using newton-HSSOR method," International Conference on Mathematical Sciences and Statistics, Kuala Lumpur, pp. 285-295, 2013.

[15] J. V. L. Chew and J. Sulaiman, "Implicit finite difference solution of one-dimensional porous medium equations using the half-sweep newton-explicit group iterative method," Journal of Engineering and Applied Sciences, vol. 13, pp. 1286-1290, 2018.

[16] A. R. Abdullah, "The four Explicit Decoupled Group (EDG) method: A fast poisson solver," International Journal of Computation Mathematics, vol. 38, pp. 61-70, 1991.

[17] J. V. L. Chew and J. Sulaiman, "Solution of one-dimensional porous medium equation using half-sweep newtonMSOR iteration," Advanced Science Letters, vol. 24, pp. 1906-1911, 2018.

[18] M. Alibubin, et al., "Half-sweep AOR iteration with rotated nonlocal arithmetic mean scheme for the solution of 2d nonlinear elliptic problems," Advanced Science Letters, vol. 24, pp. 1922-1926, 2018.

[19] A. Saudi and J. Sulaiman, "An efficient two-stage half-sweep modified arithmetic mean (HSMAM) Method for the solution of 2D elliptic equation," Advanced Science Letters, vol. 24, pp. 1917-1921, 2018.

[20] J. Sulaiman, et al., "Fourth-order solutions of nonlinear two-point boundary value problems by newton-HSSOR iteration," 3rd International Conference on Mathematical Sciences (ICMS3). Kuala Lumpur, vol. 1602, pp. 69-75, 2014.

[21] A. Hadjidimos, "Successive over-relaxation (SOR) and related methods," Journal of Computational and Applied Mathematics, vol. 123, pp. 177-199, 2000. 
[22] D. Young, "Iterative methods for solving partial difference equations of elliptic type," Transactions of the American Mathematical Society, vol. 76, pp. 92-111, 1954.

[23] A. Sunarto, et al., "Full-sweep SOR iterative method to solve space-fractional diffusion equations," Australian Journal of Basic and Applied Sciences, vol. 8, pp. 153-158, 1954.

[24] M. Deghan, "Numerical solution of one-dimensional parabolic inverse problem," Applied Mathematics and Computation, vol. 136, pp. 333-344, 2003.

[25] J. J. Benito, et al., "Solving parabolic and hyperbolic equations by the generalized finite difference method," Journal of Computational and Applied Mathematics, vol. 209, pp. 208-233, 2003.

[26] W. Liao, et al., "Direct numerical method for an inverse problem of a parabolic partial differential equation," Journal of Computational and Applied Mathematics, vol. 232, pp. 351-360, 2009.

[27] S. Gupta, et al., "Analytical solutions of convection-diffusion problems by combining Laplace transform method and homotopy perturbation method," Alexandria Engineering Journal, vol. 54, pp. 645-651, 2015.

[28] J. Sulaiman, et al., "Quarter-sweep iterative alternating decomposition explicit algorithm applied to diffusion equations” International Journal of Computer Mathematics, vol. 81, pp. 1559-1565, 2004.

\section{BIOGRAPHIES OF AUTHORS}

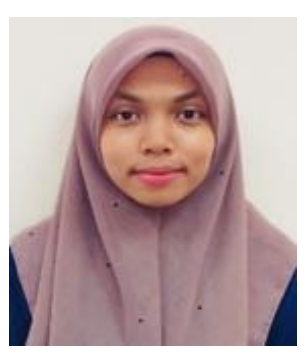

Nur Afza Mat Ali received B. Sc (Hons) in Mathematics with Economics from the Universiti Malaysia Sabah, Malaysia in 2017. She is currently a M.Sc. candidate in Applied Mathematics at the Faculty of Science and Natural Resources, Universiti Malaysia Sabah, Malaysia. Her research interest includes numerical analysis, partial differential equation, similarities solution and finite difference in which her research is currently on the iterative method with wave variable transform for solving parabolic differential equation.

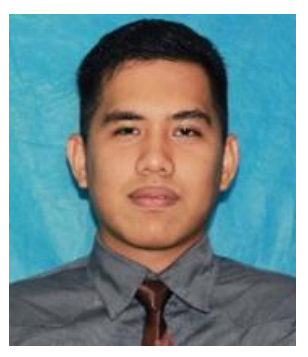

Rostang Rahman is currently studying as a M.Sc student in applied mathematics at the Faculty of Science and Natural Resources, Universiti Malaysia Sabah. He has graduated from the same university for his B. Sc (Mathematics with Economics). His current research interest is Numerical analysis for PDEs, in which his research is currently on the Caputo's finite difference solution of fractional two-point boundary value problems via iterative methods.

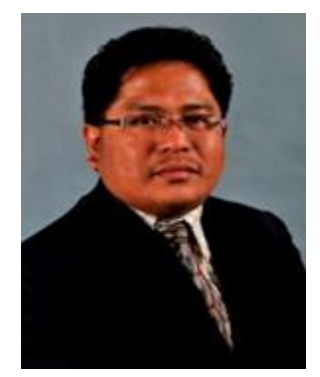

Jumat Sulaiman has received Ph.D in Industrial Computing from the Universiti Kebangsaan Malaysia (UKM), Malaysia in 2004. His current research interests are numerical analysis for PDEs, IEs and IDEs and development of mathematical models. He has published widely in numerical analysis using spline polynomial, finite element and finite difference methods, Scientific Computing (Multigrid algorithms, Points and Block Iterative Algorithms), development of nonlinear mathematical models, robot path planning and numerical analysis of image processing. In 1998, he was appointed as a lecturer at UMS, Malaysia. Again in 2005, he was appointed as a senior lecturer at UMS, Malaysia. He is currently working as Associate Professor at Universiti Malaysia Sabah (UMS), Malaysia since 2006.

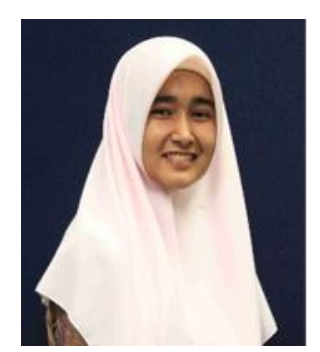

Khadizah Ghazali is a lecturer in the Faculty of Science and Natural Resources at Universiti Malaysia Sabah, where she teaches Basic Mathematics and Statistics, Calculus, Advanced Calculus, and Business Mathematics. She has a first and second degree in Mathematics from Universiti Kebangsaan Malaysia. She is currently a Ph.D. candidate in Applied Mathematics at the Faculty of Science and Natural Resources, Universiti Malaysia Sabah. Her research interests include Optimization (Large-scale Unconstrained Problems), Numerical Analysis, Partial Differential Equation, and Ethnomathematics. 